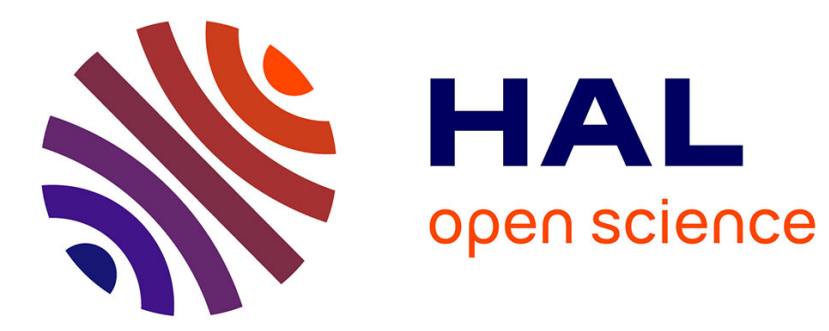

\title{
Le "contrasto" en forme de procès chez Jacopone da Todi
}

\author{
Cécile Le Lay
}

\section{To cite this version:}

Cécile Le Lay. Le "contrasto" en forme de procès chez Jacopone da Todi. Arzanà. Cahiers de littérature médiévale italienne, 2003, Les voix multiples, 9, pp.93-120. 10.3406/arzan.2003.922 . hal01541098

\section{HAL Id: hal-01541098 \\ https://hal.science/hal-01541098}

Submitted on 17 Jun 2017

HAL is a multi-disciplinary open access archive for the deposit and dissemination of scientific research documents, whether they are published or not. The documents may come from teaching and research institutions in France or abroad, or from public or private research centers.
L'archive ouverte pluridisciplinaire HAL, est destinée au dépôt et à la diffusion de documents scientifiques de niveau recherche, publiés ou non, émanant des établissements d'enseignement et de recherche français ou étrangers, des laboratoires publics ou privés. 


\section{Le contrasto en forme de procès chez Jacopone da Todi}

Cécile Le Lay

\section{Citer ce document / Cite this document :}

Le Lay Cécile. Le contrasto en forme de procès chez Jacopone da Todi. In: Arzanà 9, 2003. Les voix multiples. Du conflit au dialogue. pp. 93-120;

doi : 10.3406/arzan.2003.922

http://www.persee.fr/doc/arzan_1243-3616_2003_num_9_1_922

Document généré le 27/04/2017 


\section{LE CONTRASTO EN FORME DE PROCES CHEZ JACOPONE DA TODI}

Fn parcourant les laudes de Jacopone, on s'aperçoit - $q u$ 'un certain nombre de textes dialogués s'organisent sur le modèle du procès en justice et que par suite le champ lexical lui-même puise largement dans le monde juridique. Si le poète a choisi d'exploiter métaphoriquement un domaine aussi particulier que celui du droit et de la justice, il est intéressant pour nous de l'explorer pour mieux comprendre son message.

Tout d'abord, cette démarche a des précédents: la tradition poétique courtoise avait déjà assimilé et transposé les liens entre vassal et seigneur pour illustrer ce que pouvait être le service d'amour de l'A Amant auprès de sa Dame et développer le thème des droits et des devoirs de chacun. La Bible elle-même constituait un vaste réservoir de représentations judiciaires où Dieu intervient en tant que Juge suprême (en particulier au moment du Jugement Dernier, véritable projection imaginaire s'inspirant des procès réels).

Mais on pourrait également penser au contrasto didactique, issu de la poésie latine de l'époque (conflictus, altercatio, causa, etc.): de fait, les laudes sont destinées à instruire des novices. En choisissant de donner un cadre explicitement juridique à certaines laudes dialoguées, Jacopone renouvelle en quelque sorte ce genre littéraire, car il rétablit le lien qui existait entre le contrasto et la 
controversia de la rhétorique imperiale, dont le déroulement imitait celui d'un débat judiciaire.

De plus, à la différence de la plupart des contrasti (ou des conflictus latins), la quaestio débattue dans ces laudes n'est pas présentée comme un problème théorique : le fait qu'il existe un enjeu judiciaire (condamnation, réhabilitation ou grâce) et que la sentence du juge ait des conséquences réelles sur l'inculpé (ou suppliant) permet de se rendre compte qu'il s'agit ici d'un procès à part entière.

La vraisemblance de ces mises en scène est confortée par le lexique juridique. La précision des termes montre que, même s'il n'a jamais exercé la profession d'avoué avant son entrée chez les Frères Mineurs (aucun document ne le prouve), Jacopone s'est intéressé a l'intense activité juridique qui caractérisait la vie des Communes au XIIT siecle. Nous verrons que c'est en partie grâce à cet intérêt pour les termes techniques et les procédures judiciaires en vigueur qu'il a renouvelé la tradition littéraire et religieuse de son époque.

Enfin, une constante mérite d'être soulignée d'emblée: le juge auquel on a recours est Dieu en personne, ou JésusChrist. Les dramatisations judiciaires que Jacopone imagine se situent donc sur un plan spirituel, celui de la justice divine, ce qui permet de comprendre qu'en fait, à chaque fois, l'enjeu véritable est une vérité théologique que les novices doivent assimiler.

Parmi les différents exemples possibles, nous n'en retiendrons qu'un seul, celui de la laude 18', car elle illustre bien la façon dont Jacopone se sert de la forme du procès pour structurer sa poésie, prendre ses distances par rapport a la tradition, et renforcer l'effet de son message sur ses destinataires. 
C'est souvent l'Homme qui est assis au banc des accusés. La laude 18, au contraire, le présente en tant qu'accusateur, osant porter plainte contre Dieu lui-même : " 'Amour, Amour adoré pourquoi m'as-tu abandonné, Amour'» (v. 1). En fait, il s'agit d'un dialogue intérieur entre l'âme et Dieu, qui, sur le modele du Cantique des cantiques interprété par la mystique médiévale comme un dialogue entre le Christ (1'Epoux) et l'Eglise (1'Epouse), est objectivé ici par Jacopone sous la forme d'un litige entre mari et femme, entierement présenté au style direct. En voici le déroulement.

L'«épouse » s'adresse à son « mari » en l'appelant Amour d'une façon répétitive (en début et fin de strophe comme pour les coblas capfinidas): elle commence par lui demander d'expliquer la raison contingente (« casone ») de son départ, mais son désarroi se transforme rapidement en reproche, et elle se met a accuser son mari de l'avoir volée en partant avec son amour comme un voleur. La menace qui pèse sur ce " grand voleur " ( granne furone ») est décrite à l'aide d'une comparaison, dont le comparant est emprunté au registre juridique du droit commercial public ${ }^{2}$, et le comparé se situe à la Cour d'Amour $^{3}$, créant un lien métaphorique entre le plan de la fiction (le tribunal céleste) et le plan de la réalité (les tribunaux civils ou commerciaux). La situation paradoxale de cette accusation portée contre Celui-là même qui est sollicité pour trancher la question est ainsi présentée avec un véritable souci de vraisemblance.

L'analyse de certains termes techniques permettra par la suite de décrire avec plus de détails la réalité à laquelle Jacopone se réfere.

Au cours de la strophe suivante, qui ne figure pas dans l'édition Ageno", le comparé est entierement transposé sur un plan juridiques. De même, dans une autre strophe 
absente de l'édition Ageno, nous retrouvons la nécessité de faire appel ("archiamarsene ») a la Cour d'Amour, comme à un véritable tribunal, vu l'impossibilité d'estimer le montant du vol'.

La série d'exempla concrets que l'épouse allègue pour appuyer son accusation semble servir de preuve, comme dans un exercice de rhétorique traditionnelle ${ }^{7}$ : elle invoque ainsi le vol ordinaire , le vol d'un banquier (et son déshonneur)", le vol d'un homme d'affaires dans une compagnie (détournement de fonds) "', le refus de vendre une marchandise pourtant exposée pour être vendue"., puis elle passe d'un plan commercial public à un plan commercial privé $e^{12}$, insiste sur la comparaison avec le comportement blâmable de celui qui montrerait et refuserait la nourriture à un affame ${ }^{13}$, pour finir par l'exemple du locataire en fuite ${ }^{14}$.

La quête de l'Epoux, dont l'ascendance biblique est des plus nobles, semble tourner à une sorte de scène de ménage mais, du fait que les arguments sont étayés d'exemples empruntés au droit pénal, le litige initial prend effectivement les apparences d'une véritable plainte judiciaire ayant lieu dans une véritable cour de justice.

La maniere dont sont repris ici les artifices rhétoriques des orateurs et des prédicateurs (amplification par accumulation de comparaisons qui servent d'exempla), montre combien notre auteur est resté maître dans cet art. Il s'agit d'un réalisme de second degré qui révèle chez Jacopone le désir de prendre ses distances par rapport à une certaine culture dominante, et qui l'apparente en quelque sorte aux poètes comico-réalistes de son époque ${ }^{15}$, bien que ses objectifs soient totalement différents.

Après les seize premières strophes et l'incipit (v. 1 à 65) au long desquels était présentée la plainte de l'Homme (âme-épouse) et son argumentation solide, l'accusé (Dieu- 
époux) « défend sa cause » en cinq strophes seulement (“'brièvement je réponds'»).

Il commence par s'adresser directement à son adversaire, sans reprendre l'image de l'épouse, dévoilant ainsi que l'accusateur n'était autre que l'homme lui-même. Il conteste aussitôt le bien-fondé de l'image du " mauvais locataire ", dont le plaignant s'etait servi en dernier pour justifier son recours a la justice : $l^{\prime}$ accusé lui reproche ainsi de l'avoir chassé parce qu'il préférait héberger le “ monde " (" ce qui est contre Dieu »), alors qu'au moment où il avait décidé d'habiter avec son épouse, il avait $l^{\prime}$ intention de rester ${ }^{16}$. Logiquement, l'accusation d'injustice se retourne contre l'accusateur qui se plaint sans raison'.

Dans la strophe suivante, il affirme que l'« épouse » n'avait aucun motif de se plaindre d'être délaissée vu toutes les dépenses qu'il avait engagées pour cette demeure et tout le mal qu'il avait eu à la nettoyer ${ }^{18}$. Puis il démontre que l'accusation de vol portée contre lui est sans fondement puisque en réalité il est parti avec son bien propre ${ }^{14}$. Le contenu du vers 75 est repris et amplifié au vers 77 , avec l'apparition du mot latin " ergo » en début de vers, qui indique clairement que l'orateur est arrivé à une conclusion logique et irréfutable de son développement argumentatif, présentee ici aussi sous forme de question rhétorique ${ }^{20}$.

Les deux strophes suivantes reprennent la menace de "blâme " qui pesait sur Amour pour son refus de subvenir aux besoins de sa " femme ": dans la première, à l'aide d'un excmplum, il fait remarquer que si on prête une chose à un "vilain " incapable de l'apprécier, on ne peut être blâmé de la lui reprendre et de ne pas vouloir la lui rendre sans conditions ${ }^{21}$; puis, dans la deuxième et dernière strophe de sa plaidoirie, Amour s'adresse directement à 
son épouse et lui dit qu'elle sait bien que c'est elle qui l'a couvert de honte en le chassant hors de chez elle alors qu'il y était venu bien des fois ${ }^{22}$, de sorte qu'il peut conclure en lui posant à nouveau une question dont la formulation laisse entendre qu'il s'agit d'une hypothèse si improbable qu'elle sera obligée d'admettre que c'était lui qui avait raison en agissant ainsi $i^{23}$.

Il est en effet bien téméraire d'intenter un procès à Dieu en personne, sachant qu'il est impossible de rivaliser avec lui, même sur un plan strictement logique: Celui-ci a " plaidé sa cause » de telle manière que l'homme n'a plus qu'à s'incliner (renonçant à ses "récriminations » et à sa position de "nuque raide ", typiques du peuple d'Israël dans l'Ancien Testament) pour subir le châtiment divin'-4.

Comme le rappelle Peter Stotz dans son analyse de la double origine du conflictus dans la littérature latine du Moyen Âge, le fait de choisir "une construction en blocs" plutôt qu'un "rapide échange de répliques en vers" renvoie clairement à l'origine "rhétorico-juridique liée à la pratique du barreau ", sur le modele du débat devant un juge, et non au modèle de la compétition bucolique:" Cette laude 18 se sert donc d'une structure de procès simplifiée (Amour est à la fois l'accusé, la défense et le juge), mais bien codifiée: la série d'exemples invoqués à l'appui de l'accusation en est la preuve, ainsi que la concision de la réponse de l'accusé.

Par ailleurs, la supériorité de l'argumentation d'Amour sur celle de son adversaire se révèle de façon probante dans l'efficacité du style concis: Jacopone donne ainsi l'exemple à suivre grâce à un procès fictif qui illustre l'opposition entre deux types de discours et la victoire du plus bref. Nous reconnaissons là ce que Franco Suitner considere comme étant l'une des qualités qui prouvent sa fidélité aux enseignements de saint François ${ }^{2 n}$. 
Il s'agit donc de références suffisamment claires pour permettre aux lecteurs de comprendre facilement les caractéristiques et les enjeux de la tentation qui guette tout croyant lors d'une expérience de sécheresse intérieure: la tentation de se répandre en invectives inutiles contre Celui qui est la fidélité même et n'attend rien d'autre qu'un signe d'humilité de la part du plaignant pour revenir faire sa demeure en son âme ${ }^{27}$.

Loin de tourner la réalité en dérision, comme le faisaient les poètes comico-réalistes, Jacopone se moque plutôt $d$ 'une certaine littérature religieuse pompeuse et sclérosée, et cherche à redonner force et vraisemblance au dialogue avec Dieu. A ce propos, Giovanni Getto parle d'un " réalisme d'expression ou de communication, un réalisme verbal, pour lequel le poète, poussé par une donnée suprasensible, dans sa volonté de la traduire en des termes compréhensibles, a recours à une réalité physique " ${ }^{28}$. Puis il ajoute que ce "réalisme verbal se concrétise sous de multiples formes ". L'une de ces formes consiste à mettre en scène des procès fictifs. Nous voyons ainsi à quel point Jacopone est capable de décrire les réalités spirituelles avec la plus grande vraisemblance, afin d'éveiller l'intérêt de ses lecteurs.

La conclusion du litige laisse apparaître une autre dimension de la justice divine, qui permet une réconciliation pleine et entiere entre l'âme et Dieu: nous y reviendrons par la suite.

En nous arrêtant à présent sur certains termes juridiques plus spécifiques, nous chercherons à préciser les motivations culturelles qui ont guidé Jacopone dans ses choix linguistiques et littéraires, et nous verrons que cette richesse figurative et lexicale représente un autre aspect du " réalisme verbal » dont il se sert pour clarifier son message. 
Nous chercherons d'abord à analyser certains termes spécifiques de la littérature courtoise, puis d'autres qu'il puise dans un registre juridique contemporain.

Parmi les topoi juridiques de la littérature courtoise présents chez Jacopone, Piero Cudini a relevé la présence de la «Seigneurie » $d^{\prime}$ Amour ${ }^{29}$. Après avoir donné quelques exemples tirés de l'Ecole sicilienne, il cite, entre autres, les vers 46-48 de la laude $18^{31}$. Même si la référence à la métaphore courtoise est probablement implicite, le passage cité permet en fait d'évoquer à la fois une cérémonie $\mathrm{d}^{\prime}$ « hommage » telle qu'elle pouvait encore exister au temps de Jacopone et un engagement matrimonial qui implique pour l'époux la prise en charge des dépenses de l'épouse. Le réalisme de la description l'emporte ainsi sur toute allusion à la littérature courtoise puisqu'il s'agit de décrire la solidité des liens juridiques qui unissent l'âme et Dieu, en renforçant la métaphore des liens matrimoniaux sur laquelle est bâtie toute la laude.

Nous retrouvons plus loin la présence de la Seigneurie d'Amour quand apparaît la Cour d'Amour, dans un contexte que nous avons déjà analysé pour le réalisme juridique des comparaisons alléguées comme preuves à charge par l'epouse-accusatrice.

Au lieu d'être conservée dans une sphère de conventions d'écriture, la métaphore courtoise de la Seigneurie d'Amour est donc "resémantisée " ${ }^{31}$ grâce à de nouvelles métaphores juridiques empruntées au monde réel. Il faut cependant préciser que cette transposition du Service d'Amour sur un plan religieux est facilitee par le fait que le langage religieux était déjà calqué sur le langage féodal (voir les expressions courantes: Notre Seigneur, NotreDame, etc.).

La fin de cette laude offre un autre exemple de cette transformation lorsque, après la manifestation du repentir de son épouse, Amour justifie son retour inesperé en 
précisant qu'il ne veut pour rien au monde être accusé $d^{\prime}$ 'infidelite $e^{32}$. On trouve en effet le même terme «fallanza » chez les Siciliens, ou chez des auteurs de poésie courtoise comme Chiaro Davanzati ou Dante da Maiano, appliqué a la relation adultère de l'amour courtois, alors que chez Jacopone il renvoie explicitement à la trahison de l'engagement contracté lors d'un mariage ${ }^{33}$.

Le fait de qualifier cet amour de "légal " situe la trahison sur un plan moral et juridique puisque le sens de "loyal " qu'Ageno lui attribue ne contredit en rien l'idée qu'il s'agit ici d'un serment à respecter ${ }^{24}$. Il est alors évident que dans le cas où un tel pacte est rompu, on peut craindre un recours en justice : le " [fare] lamentanza " de la part d'un " pare » ".5.

Nous reviendrons plus loin sur ces liens métaphoriques entre Dieu et sa créature qui reprennent l'image d'un engagement à la fois féodal et nuptial, dont la rupture aurait des conséquences pénales que Dieu luimême préfère éviter.

Mise à part l'évidente " transposition d'Amour du plan terrestre au plan divin " ${ }^{3 \circ}$, qui pourrait également se référer à la Justice et qui permet de redonner vie à certaines expressions de la poésie courtoise, nous avons vu qu'il est aussi possible de déceler une technique de transformation opposée, comme l'avait déja fait remarquer Piero Cudini ${ }^{27}$, lorsque Jacopone exprime les concepts les plus sublimes au moyen d'un lexique apparemment incompatible avec ces notions.

On peut en effet relever dans certaines laudes " une série de termes techniques [juridiques] qui sont laissés de côté, à cause d'une pauvreté linguistique évidente, par les poètes 'provinciaux' [...] et, à cause d'une restriction du vocabulaire consciente, par les représentants des grands courants 'tragiques' $)^{36}$. 
Ainsi, dans la laude 18, la description de la Cour d'Amour est revitalisée dans un sens spécifiquement juridique, grâce à l'introduction d'une comparaison que nous avons déjà relevée, où le comparant ${ }^{20}$ introduit un terme technique très concret, le verbe "pigliare ", qui signifie " capturer, arrêter ». Le comparé transpose alors sur un plan métaphorique ce même réalismet".

La strophe suivante, que l'on trouve uniquement dans l'édition Mancini, réunit les deux plans de la métaphore, comme si l'accusateur pouvait réellement "pigliare" Amour". D'autres termes techniques illustrent ici cette description : la " carta de la presaglia " est un mandat d'arrêt qui permet de se saisir du coupable, quel que soit l'endroit où il se trouve $e^{+2}$ (elle s'identifiait à l'époque avec la " carta della rappresaglia " ${ }^{+3}$ ), et le " cortese " est le " capitano di giustizia " " qui préside la cour de justice.

A ce propos, Robert Davidsohn souligne l'importance du problème des "rappresaglie " pour le commerce médiéval, en rappelant qu'une longue tradition permettait qu'elles s'appliquent à tout ressortissant de la même commune que le coupable : l'origine de la "Mercanzia " a Florence a été déterminée par le souci de défendre les marchands florentins contre une telle pratique et de réglementer le commerce pour le rendre plus sûr".

Le fait que Jacopone parle de " carta de la presaglia " prouve qu'il s'agit ici d'une forme déjà réglementée de cet usage et que le "grand trésor"volé par Amore à son épouse justifie que l'on prenne une telle décision a l'encontre de ce «grand voleur».

Dans l'autre strophe ajoutée par Franco Mancini, l'épouse renchérit en affirmant qu'elle doit faire appel au tribunal car les dommages qu'elle a subis ont une valeur inestimable ${ }^{\text {ti. }}$. Le champ sémantique du verbe voler, "furare ", s'élargit grâce à des synonymes tels que " sot- 
trarre », " rapire » et « derobare », tandis que les références au droit commercial se multiplient dans les différentes comparaisons qui servent à étayer les accusations.

Même en se limitant à celle qui compare le vol $d^{\prime}$ Amour à celui que commettrait un banquier de renom ${ }^{47}$, on peut mettre en évidence le fait qu'il s'agit d'un abus de confiance ${ }^{48}$, et que cette circonstance aggravante a des conséquences dramatiques : en effet, ce banquier est voué au déshonneur lorsque l'affaire est divulgué ${ }^{49}$, et dès lors plus personne ne voudra lui confier son argent ${ }^{51}$.

La consonnance courtoise qui réapparaît dans les mots-clés de ce passage (" nomenanza » et "dubitanza ») prouve que cette référence littéraire est presque obligatoire dès qu'il s'agit d'honneur et de confiance, mais ici aussi Jacopone lui redonne un sens plus concret grâce au réalisme du contexte.

En effet, on peut se rendre compte du poids réel de cette comparaison si on considere l'importance de la réputation dans la vie communale: dejà, d'après Robert Davidsohn, la seule suspicion publique suffisait pour contraindre un juge a poursuivre une procédure judiciaire ${ }^{51}$, et dans le cas d'un débiteur insolvable, le créancier pouvait même demander que celui-ci soit banni de la ville ${ }^{52}$. De plus, lorsqu'a Florence les « Arts » élargissent leur juridiction (pendant les trente dernières années du XIII" siècle et au début du XIV"), on constate que le fait même d'être exclu d'une corporation « ne signifiait rien moins qu'une déclaration publique d'infamie et la ruine économique ", et $q u^{\prime}$ une " telle exclusion pouvait être appliquée comme una véritable sanction pénale " ${ }^{53}$. Enfin, il fait encore allusion à la renommée à propos des "peintures infamantes " ${ }^{54}$, qui constituaient une véritable mesure de rétorsion pénale contre les réfractaires ${ }^{55}$ et possédaient, semble-t-il, une force de dissuasion efficace ${ }^{56}$. 


\section{CéCILE LE LAY}

L'affaire est donc présentée de telle sorte que le plan métaphorique semble disparaître au profit d'une réalité juridique qui multiplie les allusions à la vie telle que la connaissaient les destinataires de l'époque. Ainsi, grâce à ce type d' " inserto espressionistico ", Jacopone montre son refus de "faire allégeance » à un code lyrique devenu trop abstrait $^{57}$ : il préfère exprimer crâment tous les griefs que l'homme peut avoir contre Dieu.

En conclusion de ce bref aperçu lexical, qui visait a déterminer l'origine et les contaminations des differents registres juridiques utilisés par Jacopone, nous pouvons confirmer l'existence de ce que Marco Santagata décrit comme la "désinvolture avec laquelle Jacopone manie res et verba extralittéraires " ${ }^{i x}$. Il est par ailleurs possible de deviner que sa véritable source d'inspiration réside dans son expérience mystique personnelle et que ses préoccupations principales se situent à la fois sur un plan pedagogique et spirituel. De ce fait, rien de ce qui se dit n'est à exclure a priori du message poétique, du moment que "l'effet de réel » obtenu peut favoriser la compréhension et l'adhésion du plus grand nombre.

Toujours dans le but de se montrer encore plus convaincant, Jacopone utilise souvent un procédé rhétorique très répandu à son époque: la personnification. De ce point de vue, la laude 18 est particulière, dans la mesure où elle est apparemment centrée sur une seule personnification, celle d'Amour. Cependant, elle se présente sous la forme $d^{\prime}$ un litige entre mari et femme, dont le caractère symbolique n'est dévoilé qu'à la fin, lorsque Amour répond aux accusations de son épouse en l'appelant «om ». Cet homme-épouse se présente ainsi comme une autre personnification : celle de l'âme humaine. 
En définitive, même si le recours aux personnifications pouvait être devenu (ou risquait de devenir) un simple expédient stylistique, pour Jacopone il correspond à un véritable instrument pédagogique dont le maniement confirme son étonnante " capacité à synthétiser et à exprimer avec une très grande clarté et avec précision des concepts compliqués et difficiles $"{ }^{54}$.

Ainsi, du fait qu'il a souvent recours à cette technique que Franco Suitner appelle a juste titre "teatralismo " ${ }^{\text {" }}$, Jacopone peut être considéré comme l'un des précurseurs des laudes dramatiques et de la représentation sacré $e^{f 1}$.

L'angle d'approche spécifique de notre étude a donc permis de montrer comment Jacopone utilise ses competences littéraires, théologiques et juridiques pour crér de véritables mises en scènes capables d'augmenter l'effet de son message sur ses lecteurs. Nous pouvons cependant préciser avec André Pézard qu' "il ne se pique ni d'art ni de littérature; il cherche seulement à convertir ses frères; tout autre souci doit être à ses yeux coupable vanite " ${ }^{62}$.

Or, du fait qu'une part importante de ce message poétique traite de la justice divine, le moment est venu d'analyser la façon dont elle se présente et la signification particulière qu'elle prend pour Jacopone.

La justice divine se manifeste tout d'abord dans le fait que le juge auquel on a recours est Dieu en personne: étant donné que Jacopone s'adresse principalement à des novices, nous comprenons en effet que, pour lui, seule la référence au Juge suprême est vraiment digne d'intérêt.

Cependant, le début de notre étude a montré à quel point le poète est capable de donner du relief à ses mises en scène de procès, grâce aux rapprochements opérés 
volontairement entre la réalité fictive de ses laudes, le système juridique en vigueur à son époque, et certains éléments de la tradition lyrique et rhétorique.

En fait, nous voyons que cela permet à Jacopone d'introduire ses lecteurs dans le mystère d'une réalité divine qui n'est pas seulement intervenue au moment de la création de l'homme, mais qui continue à agir sur la destinée humaine, selon des modalités qui semblent compréhensibles par certains aspects, tout en restant inevitablement hors d'atteinte pour l'esprit humain.

Ainsi, au lieu de dispenser un enseignement théologique abstrait sur les caractéristiques de la Justice divine, il se place sur un plan concret, en cherchant à montrer les enjeux qui sont à l'origine de l'intervention de Dieu dans la vie de l'homme. Et pour lui, en tant que poète, la meilleure façon de présenter ces enjeux consiste à mettre en scène un débat entre l'homme et Dieu.

Effectivement, la critique s'est aperçue que Jacopone est l'un des poètes qui a manifesté le plus intensément sa volonté de pénétrer le mystère de la nature divine, et que la forme du débat était celle qui lui permettait le mieux d'exprimer ce désir. Cette tension entre les exigences de la raison humaine et celles de la volonté divine l'habitait si profondément qu'on a pu dire de lui qu'il était « le mystique, et peut-être le poète, du contrasto, de la rencontre et du choc de ces deux dimensions, humaine et divine, une rencontre qui produit une espèce de déséquilibre et de saut " ". Ce saut qualitatif n'est autre que l'intervention de la grâce divine, qui se refletera dans la récurrence du mot " esmesuranza » chez Jacopone (en contraste avec l'idéal d'équilibre de la poésie courtoise).

Cette capacité de rendre compte de la nature divine d'une façon plus imagée qu'analytique correspond à ce que, à la même époque, saint Bonaventure avait souligné 
dans ses recherches philosophiques: à la différence de saint Anselme, qui se base essentiellement dans ses réflexions sur le fait que la notion logique de Dieu est présente en l'homme, il insiste plutôt sur le fait que l'image de Dieu est déjà imprimé dans l'âme humaine, et que la grâce divine peut l'elever, par paliers successifs, des vérités les plus immédiatement perceptibles à celles qui sont éternelles et transcendantes (voir l'Itinerarium mentis in Deum). La logique des accusations de l'epouse ne peut effectivement pas rivaliser avec celle $d$ 'Amour, mais, devant la sincérité du repentir de son accusatrice, celui-ci préfère clore le débat en décidant, d'une façon totalement inespéree, comme l'est toute intervention de la grâce divine, de réintégrer les lieux dont il avait été chassé.

Parmi les nombreuses laudes que nous pouvons étudier, la laude 18 est donc l'une de celles qui permettent le mieux de montrer jusqu'où Jacopone est capable de pousser l'intensité du dialogue entre l'homme (l'âmeépouse) et Dieu (Christ-epoux). Cette laude fait ainsi partie de " ces textes dans lesquels le dialogue entre le pénitent et la divinité se déroule de façon directe, et avec une certaine hardiesse ", sans doute grâce à l'arrivée de " la nouvelle spiritualité franciscaine qui favorise et en quelque sorte exige un rapport avec la divinité de type spontané et direct, éloigné de la solennité qui s'exprimait si bien dans la tradition figurative byzantine ${ }^{64}$, de même que dans une certaine tradition lyrique ou religieuse devenue stéréotypée, pourrions-nous ajouter.

Jacopone invente ainsi une forme originale de tenson fictive, dans le cadre d'une poésie religieuse qui a aussi une vocation éducative, forme qui engendrera comme nous l'avons dit des textes dramatiques. 


\section{CécIle Le Lay}

\section{ANNEXE \\ Lauda 18 \\ (extraits)}

«Amor, diletto Amore, perché m'ài lassato, Amore?

Amor, di'la casone de lo to partemento, ché $\mathbf{m}$ 'ài lassata afflitta en gran dubetamento; se da schifezza èi vénto, vogliolten'satisfare; $s^{\prime} e^{\prime} m e$ ' $n$ voglio tornare, non te ne torne, Amore?

|... Amore, omo che fura ad altri gran tesoro, 14 la corte, poi lo piglia, fali far lo restoro;

'nanti a to corte ploro che me faccia rasone de te, granne furone, che m'ài sottratto amore.

[ La carta de la presaglia mo me ne fa el cortese ch'eo te pozza pigliare en qual te trovo paiese; no n'aio da far le spese, morraio ne la strata, che tal ricchezza ài furata ch'e'me nne mogo, Amore.]

A more, lo mercatante, ched è multo apreiato, e 'n ascus' $i$ fa 'l sottratto a chi i ss'è tutto dato, da poi ch'è spaliato, perde la nomenanza e onn'om n'à dubitanza de crederseli, Amore.

Amor, li mercatanti c'à fatta compagnia e l'un fa li sottratti, non li sse par che sia; tutta moneta ria lassa ' $n$ lo so taschetto, la bona tutta à scelto; sì ne rapisce, Amore.

|... | A mor, omo ch'è ricco et à moglie ennarrata, tornali a granne onore, s'ella va mennicata? Rechez'à'esmesurata e non sai quella te 'n fare e poimen'satisfare e non par che ' 1 facci, Amore. 


\section{LE CONTRASTO EN FORME DE PROCĖS}

Amor tu èi meo sposo, àime per moglie presa; tórnate a granne onore vetata m'è la spesa? Sommete en mano mesa et aime en le to mane; la gente sprezzata m’àne, sì so'denigrata, Amore.

[...] Amor, om c'à abbergato et à 'l tolto a pesone, se 'l larga 'nant' el tempo, que no vòl la rasone c'arturne a la masone e pagi tutta la sorte? Ià non vol cose torte; $a l<e>i$ me nn'archiamo, Amore ".

"Omo che te laminti, breve mente responno; tollenno lo to abbergo, crìsice far soiorno; abbergastic' el mondo e me 'n caciasti via; donqua fai villania, se tu murmuri de Amore.

Tu sai, mentr'e'ce stetti, quigne spese ce fice; non 'n te pòi lamentare, si te ne satisfice; cà ad nettarlo me misi, ch'era pleno de loto; e ficel tutto devoto per avetarce Amore.

Quanno eo me nne partîci Amor me 'n portai 'l mio; como lo pòi tu dire ch'eo me 'n portasse el tio?

Tu sai ch'ell'è sì rio c'a me non è 'n placere; ergo, co'1 pòi tu dire ch'e' te 'l tollesse Amore?

Quanno neiuna cosa ad alcuno è sprezzata e no i la renn'entrasatto, no dé'essar blasmata, s'i l'artolle a la fiata, sènno colui villano, che non sente de mano de que li à prestato Amore.

Tu sai molte fiate sì ce so' abbergato e sai co'a gran vergogna sì me nn'ài for cacciato; forsa che t'è ad grato ch'eo cce deia avetare, faccenno detoperare sì nobelissimo Amore? ». 
"Amor, ditt'ài tal scusa ch'ella se pò bastare a lo mormoramento $c^{\prime}$ aio voluto fare; voglio' 1 capo enclinare che nne facci vendetta, e non me 'n tener plu afflitta de celarmette, Amore".

«Rennendoten' pentuta, sì cce voglio artornare, 90 ancor me fusse fatto villano allecerare; non voi'che mai to pare facesse lamentanza ch'eo facesse fallanza de lo legale amore ».

\section{TRADUCTION}

«Amour, Amour adoré pourquoi m'as-tu abandonné, Amour?

Amour, dis-moi la cause de ton éloignement, car $t^{\prime} \mathrm{m}^{\prime}$ as laissée affligée en grand trouble, si tu es vaincu par le dégoût je veux te satisfaire: si je reviens sur mes erreurs reviendras-tu, Amour?

[...] Amour, un homme qui vole à un autre un grand trésor,

la cour le saisit puis le fait rembourser, devant ta cour j'implore qu'on me fasse justice, de toi, grand voleur qui $\mathrm{m}^{\prime}$ as soustrait amour.

[Le mandat d'arrêt le juge va me le faire pour que je puisse t'attrapper en quelque lieu que tu sois ; je n'en paierai pas les frais: je mourrai en chemin, 20 car tu as volé tel trésor que j'en meurs, Amour.|

Amour, le banquier qui est très estimé et vole en cachette celui qui s'en est entièrement remis à lui, après qu'il est découvert il perd sa renommée, chacun se garde de lui faire confiance, Amour. 
Amour, les marchands qui ont fait une société, et l'un d'entre eux vole sans qu'on sache qui c'est: toute la fausse monnaie il laisse dans la bourse, il a choisi toute la bonne; ainsi tu me voles, Amour. 29

[...] Amour, un homme qui est riche et a pris une épouse,

lui revient-il à grand honneur si elle va en mendiante? Tu as une richesse démesurée et tu ne sais qu'en faire, tu pourrais $m^{\prime}$ en satisfaire et il $n^{\prime}$ apparaît pas que tu le fasses, Amour.

Amour, tu es mon époux tu $\mathrm{m}^{\prime}$ as prise pour épouse, te revient-il à grand honneur que me soit refusée la dépense? Je me suis remise à ton pouvoir et tu me tiens en tes les gens m'en ont méprisée et je suis dénigrée,

mains, Amour.

[...] Amour, un homme qui a un logis

et l'as pris en location,

s'il le laisse avant le temps, que demande la justice?

$\mathrm{Qu}$ 'il retourne à la maison et paye tout ce qu'il doit? Elle ne veut chose injuste, j'en appelle à elle,

Amour. »

«Homme qui te lamentes, brièvement je réponds: en venant loger chez toi je pensais y faire séjour, mais tu y as abrité le monde et tu m'en as chassé, donc tu te montres injuste si tu te plains d'Amour.

Tu sais, quand j'y étais, quelles dépenses j'ai faites, 70 tu ne peux te plaindre tant je $t^{\prime}$ ai satisfait, j'entrepris de le nettoyer car il était plein de boue, je l'ai fait tout propre pour y habiter, Amour. 
Quand j'en suis parti j'ai emporté mon bien, comment peux-tu dire que j'ai emporté le tien?

Tu sais qu'il est si mauvais qu'à moi il ne plaît pas, ergo, comment peux-tu dire qu'Amour te l'a volé?

Quand une chose est méprisée par quelqu'un, et qu'elle ne lui est pas rendue sans conditions, il ne doit pas la blâmer

si elle lui est parfois reprise, puisqu'il est rustre 80 et qu'il n'apprécie pas aussitôt ce qu'A mour lui a prêté.

Tu sais que maintes fois j'y ai habité, et tu sais comment à grande honte tu m'en as chassé, sans doute désirerais-tu que j'y demeure sachant que tu vas blâmant ce très noble Amour? ".

"Amour, ce que tu as dit à ta défense peut satisfaire à la plainte que j'ai voulu porter; je veux baisser la tête, pour que tu me punisses; ne me laisse plus dans l'affliction en te cachant, Amour ».

"Puisque tu te repens je veux donc revenir, 90 bien que j'aie reçu vilainement congé, je ne voudrais pas qu'un de tes semblables puisse se que j'aie trahi un loyal amour ». plaindre 


\section{LE CONTRASTO EN FORME DE PROCÈS}

\section{NOTES}

1. Laude 18: «Amor, diletto Amore, perché m’ài lassato, Amore »: IACOPONE da TODI, Laude, éd. par F. Mancini, Rome-Bari, Laterza, 1974, p. 52-55.

On pourrait en ajouter quelques autres: laude $1:$ : La Bontate se lamenta ", laude 3: "L'omo fo creato vertüoso ", laude 8: «La Veretate plange, ch'è morta la Bontate», laude 63: «O lesù Cristo pietoso » etc.

2. "Amore, omo che fura ad altri gran tesoro,/la corte, poi lo piglia, fali far lo restoro'» ( $"$ 'Amour, un homme qui vole à un autre un grand trésor, / la cour le saisit puis le fait rembourser'»: v. 14-15).

3. "' "Nanti a to corte ploro che me faccia rasone/de te, granne furone, che m'ài sottratto amore'» («'Devant ta cour j'implore qu'on me fasse justice,/de toi, grand voleur qui $\mathrm{m}^{\prime}$ as soustrait amour'»: v. 16-17).

4. IACOPONE da TODI, Laudi. Trattato e Detti, éd. par F. Ageno, Florence, Le Monnier, 1953: laude LXVII, p. 277-280.

5. "'La carta de la presaglia mo me ne fa el cortese/ch'eo te pòzza pigliare en qual te trovo paiese'" ("'Le mandat d'arrêt le juge va me le faire/ pour que je puisse t'attraper en quelque lieu que tu sois'»: v. 18-19). Nous reviendrons sur ces vers pour leur analyse lexicale.

6. «'El meo danno ho pensato, ch'e'no 'I pòzzo estimare,/ déiomene archiamare a la tua corte, Amore'" ( «'J'ai pensé à cette perte, je ne peux l'estimer,/et je dois en appeler à ton tribunal, Amour'»: v. 32-33).

7. Par la suite, six exemples sont d'ailleurs présentés sous forme de questions rhétoriques (vers $35,43,47,51,59$ et 64), comme si la réponse était évidente.

8. "'La mente mea esmarruta va chidenno ' 1 dolzore,/ ché li è furato ad ore'» ("Mon esprit égaré va cherchant la douceur, / car elle lui a été dérobée'»: v. 12-13). 
9. «'Amore, lo mercatante, ched è multo apreiato,/e 'n ascus' $i$ fa 'l sottratto a chi i ss' è tutto dato,/da poi ch'è spaliato, perde la nomenanza/e onn'om n'à dubitanza de crederseli, Amore'" ("A 'Amour, le banquier qui est très estimé/et vole en cachette celui qui s'en est entièrement remis à lui,/après qu'il est découvert il perd sa renommée,/ chacun se garde de lui faire confiance, Amour'»: v. 22-25).

10. "Amor, li mercatanti c'à fatta compagnia/e l'un fa li sottratti, non li sse par che sia;/ tutta moneta ria lassa 'n lo so taschetto,/la bona tutta à scelto; si ne rapisce, Amore'» ("Amour, les marchands qui ont fait une société,/et l'un d'entre eux vole sans qu'on sache qui c'est:/ toute la fausse monnaie il laisse dans la bourse, / il a choisi toute la bonne; ainsi tu me voles, Amour'»: v. 26-29).

11. "'Amor, om c'à mercato e vendel volunteri,/vedennol quel che 'I brama, déne da lu'fugire?' " ("Amour, un homme qui a une marchandise qu'il vendrait volontiers, / en voyant celui qui la désire doit-il fuir loin de lui ?'”: v. 34-35).

12. " 'A mor, omo ch'è ricco et à moglie ennarrata/tornali a granne onore, s'ella va mennicata?'» (“'Amour, un homme qui est riche et a pris une épouse, / lui revient-il à honneur si elle va en mendiante?'»: v. 42-43); “'Amor tu èi meo sposo, àime per moglie presa;/ tòrnate a granne onore vetata m'è la spesa?'» («'Amour, tu es mon époux, tu m'as prise pour épouse, / te revient-il à grand honneur que me soit refusée la dépense?'»: v. 46-47).

13. "Amor, om che mustrasse lo pane a l'afamato/e no ne i vòlse dare, or non siria blasmato?'» («'Amour, qui montrerait un pain à l'affamé/et ne voudrait le lui donner ne serait-il blâmé ?'»: v. 50-51); “'Amor, lo meo coraio sì l'ài stretto legato,/vòlilo far perire, che li ài 'l cibo celato?'» ("Amour, mon coeur tu l'as lié si serré,/ veux-tu le faire périr, que tu lui as caché la nourriture ?'»: v. 58-59).

14. “'A mor, om c'à albergato et à ' 1 tolto a pesone,/ se '1 larga 'nant'el tempo, que no vòl la rasone/c'arturne a la masone e pagi tutta la sorte ?'» («'Amour, un homme qui a un logis et l'as pris en location,/s'il le laisse avant le temps, que 


\section{LE CONTRASTO EN FORME DE PROCES}

demande la justice ?/Qu'il retourne à la maison et paye tout ce qu'il doit?'»: v. 62-64).

15. « Con il 'realismo'di Iacopone ci siamo portati all'estremo opposto rispetto ai rimatori provinciali dai quali siamo partiti. Se per quei poeti estemporanei è la scarsa padronanza del mezzo espressivo a togliere evidenza realistica al vissuto che pure li muove a scrivere, con lacopone è il rifiuto di un codice bene appreso e da tempo dominante ad aprire la via all'inserto espressionistico. E'un po' la stessa condizione che caratterizza poeti comico-realistici fra Due e Trecento. In un realismo di secondo grado come il loro c'è spazio anche per il lessico e le immagini delle pratiche di giustizia »: $M$. SANTAGATA, "Torture e processi d'amore nella lirica italiana ", in AA. VV., Torture, tortures: débats et représentations (Actes du colloque organisé à l'occasion du 2ème centenaire de la mort de Cesare Beccaria, Université de Nancy II, 24-25 novembre 1994), p. 89.

16. “'Omo che te laminti, breve mente responno;/tollenno lo to abbergo, crisice far soiorno;/abbergastic'el mondo e me 'n caciasti via'» («'Homme qui te lamentes, brievement je réponds:/en venant loger chez toi je pensais $y$ faire séjour,/ mais tu y as abrité le monde et tu m'en a chassé' " : v. 66-68).

17. «'Donqua fai villania, se tu murmuri de Amore'» («'Donc tu te montres injuste si tu te plains d'Amour'»: v. 69).

18. “'Tu sai, mentr'e' ce stetti, quigne spese ce fice;/ non 'n te pòi lamentare, sì te ne satisfice,/ cà ad nettarlo me misi, ch'era pleno de loto;/ e ficel tutto devoto per avetarce Amore' " («'Tu sais, quand j'y étais, quelles dépenses j'ai faites,/ tu ne peux te plaindre tant je $t^{\prime}$ ai satisfait, / $j$ ' entrepris de le nettoyer car il était plein de boue,/je l'ai fait tout propre pour $y$ habiter, Amour'»: v. 70-73).

19. «'Quanno eo me nne partîci Amor me 'n portai 'l mio;/ como lo pòi tu dire ch'eo me 'n portasse el tio?'» ( “'Quand j'en suis parti $j$ 'ai emporté mon bien,/ comment peux-tu dire que $j$ 'ai emporté le tien ?'»: v. 74-75). 
20. «'Ergo, co"1 pòi tu dire ch'e'te 'l tollesse Amore?'» («'Ergo, comment peux-tu dire qu'Amour te l'a volé ?'»: v. 77).

21. "Quanno neiuna cosa ad alcuno è sprezzata/e no i la renn'entrasatto no dé'essar blasmata,/s'i l'artolle a la fiata, sènno colui villano,/ che non sente de mano de que li à prestato Amore'" ("Quand une chose est méprisée par quelqu'un, / et qu'elle ne lui est pas rendue sans conditions, il ne doit pas la blâmer/si elle lui est parfois reprise, puisqu'il est rustre/et qu'il n'apprécie pas aussitôt ce qu'A mour lui a prêté $»$ : v. 78-81).

22. "'Tu sai molte fiate sì ce so'abbergato/e sai co'a gran vergogna sì me nn'ài for cacciato'» (“'Tu sais que maintes fois j'y ai habité,/ et tu sais comment à grande honte tu $\mathrm{m}^{\prime}$ en as chassé'»: v. 82-83).

23. "Forsa che t'è ad grato ch'eo cce deia avetare,/facenno detoperare sì nobelissimo Amore?'» («'Sans doute désireraistu que j'y demeure/ sachant que tu vas blâmant ce très noble A mour?'»: v. 84-85).

24. "'Amor, ditt'ài tal scusa ch'ella se pò bastare/a lo mormoramento c'aio voluto fare;/voglio 'l capo enclinare che nne facci vendetta"» ( 'A'Amour, ce que tu as dit à ta défense peut satisfaire/à la plainte que j'ai voulu porter;/je veux baisser la tête, pour que tu me punisses'»: v. 86-88).

25. P. STOTZ, «Conflictus. Il contrasto poetico nella letteratura latina medievale ", in AA. VV., Il genere "tenzone » nelle letterature romanze delle origini, éd. par $\mathrm{M}$. Pedroni et $\mathrm{A}$. Staüble, Ravenne, Longo, 1999, p. 172.

26. « $[\ldots]$ come dice Iacopone che è un nemico dei discorsi troppo lunghi e contorti, prima di parlare è bene che l'uomo rifletta se quel che vuol dire è effettivamente utile, e quando ha deciso di farlo è bene sia conciso, in quanto la longa materia/sòl generar fastidia,/el longo abriviare/sole l'om delettare' $(65,5-8)$. Anche in questo, egli è in linea col maestro Francesco, che nella regola raccomandava ai predicatori la concisione, perché il Signore disse parole brevi sulla terra (Reg. bull., IX) ", F. SUITNER, lacopone da Todi. Poesia, mistica, rivolta nell'Italia del medioevo, Rome, Donzelli, 1999, p. 99-100. 


\section{LE CONTRASTO EN FORME DE PROCĖS}

27. Depuis le $X^{e}$ siècle, le préambule à la communion reprend la formule d'humilité confiante du centurion de Capharnaüm (Mt 8,8$)$, où apparaît clairement l'image de l'âme qui implore Dieu en reconnaissant $q \mathbf{q}^{\prime}$ elle $n^{\prime}$ est pas digne de le recevoir sous son toit: «Domine, non sum dignum [...] sub tectum meum $[\ldots] »$.

28. G. GETTO, Il realismo di Jacopone da Todi, in « Lettere italiane », VIII (1956), p. 237 (à présent in Letteratura religiosa dal Due al Novecento, Florence, 1967).

29. « Si può ancora considerare un tema centrale - quasi un topos - della poesia siciliana, che ricompare continuamente nelle 'laude erotiche'di Jacopone, il tema secondo cui Il poeta è nella signoria d'Amore», P. CUDINI, «Contributo a uno studio di fonti siciliane nelle laude di Jacopone da Todi », in « Giornale Storico della Letteratura Italiana », vol. CXLV, 1968, p. 565-566.

30. "Amor tu èi mio sposo, àime per moglie presa;/tòrnate a granne onore vetata $m^{\prime}$ è la spesa? / Sòmmete en mano mesa et àime en le tuo mane" " ("Amour, tu es mon époux tu m'as prise pour épouse, / te revient-il à grand honneur que me soit refusée la dépense? / Je me suis remise à ton pouvoir et tu me tiens en tes mains' »: v. 46-48).

31. Cf. E. LANDONI, Il " libro" e la "sentenzia". Scrittura e significato nella poesia medievale: Iacopone da Todi, Dante, Cecco Allgiolieri, Milan, Vita e Pensiero, 1990, § 3: «Risemantizzazione del lessico lirico-amoroso e capovolgimento della gerarchia di valori tradizionali ", p. 34-41.

32. «'Non voi' che mai to pare facesse lamentanza/ch'eo facesse fallanza de lo legale amore' " ("'Je ne voudrais pas qu' un de tes semblables puisse se plaindre/que j'aie trahi un loyal amour' »: v. 92-93).

33. Cf. " fallanza ", in F. MANCINI, éd. cit., " Glossario », p. 733.

34. Cf. M. CORTELAZZO, P. ZOLLI, Dizionario etimologico della lingua italiana, Bologne, Zanichelli, 1983: « legale » et « leale " ont la même étymologie latine (lex, legis) et leurs sens («légal » et « loyal ») sont difficilement dissociables. En effet, 


\section{Cécile le Lay}

" leale " est construit sur l'ancien provençal « leial » qui signifiait « légal ». Dans les textes anciens (du XII ' siècle jusqu'à Dante et au-delà) il conserve encore le sens primitif de «correspondant aux prescriptions de la loi », tout comme « legale".

35. Le contexte amène à penser qu'ici aussi il est fait allusion au droit féodal par ce mot " pare " qui peut avoir comme origine le provençal " par ", ou l'ancien français " pair ": cf. GDL.I, "pare », emploi 26.

36. P. CUDINI, op. cit., p. 569.

37. « Ed è elemento caratteristico, tipico della poesia jacoponica il cantare gli argomenti più sublimi in un tono che inoli essere non sublime, ma soltanto medio, nell'a mbito del quale può spiegarsi anche l'ibridismo lessicale e formale della lingua jacoponica, in cui vi è a un tempo presenza di termini dotti e formule retoriche latine e di espressioni rozzamente vernacolari », P. CUDINI, op. cit., p. 571.

38. M. SANTAGATA, op. cit., p. 86.

39. "Omo che fura ad altri gran tesoro, / la corte, poi lo piglia, fali far lo restoro", (" 'Un homme qui vole à un autre un grand trésor, / la cour le saisit puis le fait rembourser'»: v. 14-15).

40. " "Nanti a to corte ploro che me faccia rasone/de te, granne furone, che m'ài sottratto amore'" ("'Devant ta cour j'implore qu'on me fasse justice,/de toi, grand voleur qui m'as soustrait amour'»: v. 16-17).

41. "La carta de la presaglia mo me ne fa el cortese/ch'eo te pòzza pigliare en qual te trovo paiese'" ("Le mandat d'arrêt le juge va me le faire/ pour que je puisse $t$ attraper en quelque lieu que tu sois'»: v. 18-19).

42. M. SANTAGATA, op. cit., p. 89.

43. "Presìglin, sf. Ant. In età medievale, appropriazione legalizzata di beni di uno straniero insolvente, a titolo di rivalsa o anche arresto di un concittadino o di un compatriota debitore; rappresaglia. Carta della presaglin: carta della rappresaglia ", GDLI, vol. XIV, p. 210. Voir aussi F. 


\section{LE CONTRASTO EN FORME DE PROCĖS}

AGOSTINI, « Il volgare perugino negli « Statuti del 1342 »», in «Studi di Filologia Italiana», XXIV (1968), glossaire p. 193: "presaglia (grafia -lgla) 'rappresaglia'», avec renvoi à P. SELLA, Glossario latino italiano (Stato della Chiesa, Veneto, Abruzzi), Città del Vaticano 1944.

44. Cf. F. MANCINI, «Glossario », op. cit., p. 703. Dans la laude 63, le Diable dit à propos de lui-même qu'il est « cortese $[\ldots]$ a ccasa [sua] » (v. 57).

45. R. DAVIDSOHN, Storia di Firenze, Florence, Sansoni, 8 vol., 1957-65; vol. V (1957), parag. « Rappresaglie. Origine della Mercanzia », p. 513-518.

46. «'El meo danno ho pensato, ch'e'no 'l pòzzo estimare'» ( «'J'ai pensé à cette perte, je ne peux l'estimer' $\gg$ : v. 32).

47. «Lo mercatante, ched è multo apreiato " ("'Le banquier qui est très estimé" $"$ : v. 22).

48. "Fa 'l sottratto a chi i ss'è tutto dato » ("'Il vole en cachette celui qui s'en est entièrement remis à lui'»: v. 23).

49. «Da poi ch'è spalïato, perde la nomenanza " ("Après qu'il est découvert il perd sa renommée'»: v. 24).

50. “Onn'om n'à dubitanza de crederseli » (" 'Chacun se garde de lui faire confiance'»: v. 25).

51. R. DAVIDSOHN, op. cit., vol. V, parag. "Le corti penali", P. 582.

52. Ibid., parag. "Arresto per debiti e sbandimento », p. 510.

53. Ibid., parag. "I tribunali delle Arti », p. 539.

54. Ibid., parag. « Pittura infamante », p. 597-603.

55. Ibid., parag. « Competenza e potere punitivo dei tribunali d'Arte ", p. 550.

56. Ibid., parag. « Pittura infamante », p. 602.

57. M. SANTAGATA, op. cit., p. 89.

58. M. SANTAGATA, op. cit., p. 86.

59. F. SUITNER, op. cit., p. 90. 


\section{CécIle Le LAy}

60. "Per riuscire a condensare efficacemente nel breve giro dei suoi versi concetti e ragionamenti tanto ostici non esita a far ricorso a tutti gli artifici della sua arte letteraria e particolarmente a tutto un repertorio di espedienti 'popolari', il più importante dei quali è quel che potremmo chiamare il suo 'teatralismo'. Il suoi componimenti teologico-moraleggianti sono molto spesso 'messi in scena': vizi, virtù, attitudini, attributi della vita morale e spirituale sono spessissimo personificati, parlano e agiscono in prima persona », ibid., p. 90.

61. «[...] non sembrano dubbi i meriti di lacopone in relazione alla lauda drammatica, in una fase ben anteriore alla sua effettiva rappresentazione. [...] 'Donna del Paradiso' è l'unica lauda drammatica di cui si conosca con precisione un termine ante quem, che è poi il Natale 1306, probabile data di morte del Todino. Non è improbabile dunque che si debba proprio a Iacopone il merito primario della geniale innovazione, destinata a dar frutti tanto copiosi e tanto importanti per il teatro italiano e per l'attività letteraria in genere nel Trecento e nel Quattrocento », G. VARANINI, "Protostoria della lauda drammatica ", in Lingua e letteratura italiana dei primi secoli, vol 1, Pise, Giardini, 1994 (1 ${ }^{\text {ere }}$ publication in "Ariel », I, 1986, p. 55-70), p. 221.

62. A. PEZARD, "Faux noms de lieux dans les Laudi de Iacopone », in « Romania », LXXV, 1954, p. 486.

63. G. GETTO, op. cit., p. 236.

64. F. SUITNER, "Metamorfosi di motivi comico-giullareschi in lacopone », in AA. VV., Il genere " tenzone »..., p. 136. 